\title{
Perceived awareness, practices and perspectives on COVID-19 management among medical doctors in India: A repeated cross-sectional study
}

\author{
Saurav Basu ${ }^{1}$, Ruchir Rustagi ${ }^{1}$, Pragya Sharma ${ }^{1}$, Nandini Sharma $^{1}$, Anish Laul $^{1}$
}

\section{AFFILIATION}

1 Department of Community Medicine, Maulana Azad Medical College, New Delhi, India

\section{CORRESPONDENCE TO}

Saurav Basu. Department of Community Medicine, Maulana Azad Medical College, 2 Bahadur Shah Zafar Marg, Balmiki Basti, New Delhi, 110002, India. E-mail: saurav.basu1983@gmail.com

\section{KEYWORDS}

COVID-19, health policy, perspectives, health management, awareness

Received: 12 June 2020, Revised: 10 October 2020,

Accepted: 10 October 2020

\begin{abstract}
INTRODUCTION Medical doctors are expected to play a proactive role during the COVID-19 pandemic through effective patient management and the implementation and adherence to best practices as per the expert consensus in their country. Strengthening the self-efficacy and addressing the reasons for risk perceptions among medical doctors is essential towards successfully combating the COVID-19 pandemic. In this study, we ascertained the perceived awareness, practices and perspectives regarding the COVID-19 pandemic among medical doctors in India.

METHODS We conducted a cross-sectional study with two rounds of data collection from 3 April to 24 May 2020 among medical doctors (allopathic) and trainees (medical interns) working at any health facility across India. The data were collected using a self-administered questionnaire utilizing the Google Forms platform.

RESULTS A total of 432 medical doctors responded to the survey with 251 in the first round (3-9 April), and 181 in the second round (18-24 May). Official government sources were considered as the most authentic source of information
\end{abstract}

by most $(84.2 \%)$ participants. However, the perceived awareness on aspects of COVID-19 epidemiology, prevention and policy was inadequate in a majority (52.8\%). Moreover, $52.3 \%$ of the respondents reported never having received institutional training related to COVID-19 management protocols. The individual perceived risk of contracting COVID-19 (median score $=4$ ) was lower than the perceived threat of disease transmission to the family members (median score $=5$ ). Access to personal protective equipment (PPE) was suboptimal but it significantly improved with the progression of the pandemic $(\mathrm{p}<0.001)$.

CONCLUSIONS Significant awareness and training deficiencies related to COVID-19 were observed among medical doctors constituting a potential occupational risk hazard. The participant perspectives on public health aspects of COVID-19 management reflected heterogeneous stances, but were mostly aligned with the academic consensus. Most participants shared a direct medicalized perspective as opposed to the negative economic considerations in supporting the implementation of a nationwide lockdown.

\section{INTRODUCTION}

The COVID-19 pandemic caused by the SARS-CoV-2 virus represents a major global health crisis. In India, the second most populous country in the world, COVID-19 cases have been escalating, with the number of attributable cases and deaths increasing from 3105 and 83 respectively, on 3 April 2020, to 100326 and 3155, respectively, on 18 May $2020^{1}$.

Medical doctors are expected to play a proactive role during the COVID-19 pandemic through effective patient management and the implementation and adherence to best practices as per the expert consensus in their country. Furthermore, medical doctors are also pivotal pandemicrelated information sources for the community, and any errors in their comprehension may undermine efforts in its control. Ordinary residents during pandemics are subject to considerable anxiety and stress ${ }^{2}$, and their interactions with 
their family doctors or local clinicians can also mold their confidence and build trust in administrative efforts directed towards controlling the outbreaks. However, healthcare workers (HCWs) globally, especially medical doctors in the frontline, are exposed to a significant occupational risk of contracting the highly transmittable virus, further compounded by inadequate availability of personal protective equipment ${ }^{3,4}$. Strengthening the self-efficacy and addressing the reasons for risk perceptions among medical doctors is essential towards successfully combating the COVID-19 pandemic.

In this study, we ascertained the perceived awareness, practices and perspectives regarding the COVID-19 pandemic among medical doctors in India.

\section{METHODS}

\section{Study design, period, participants and sample size}

We conducted a cross-sectional study with two rounds of data collection of 7 days each: 1) 3-9 April 2020, and 2) 1824 May 2020; conducted among medical doctors (allopathic) and trainees (medical interns) working at any health facility across India. At 95\% confidence levels, 5\% margin of error, and expecting $50 \%$ prevalence of perceived awareness, the sample size was calculated to be 384 . The study was approved and exempted from full review by the Institutional Ethics Committee (F.1/IEC/MAMC/(73/01/2020/No49). No personally identifiable information was collected from the participants. Implied consent was applicable for the study since participation was on an opt-in and voluntary basis. Data from this study are available, on reasonable request, from the corresponding author.

\section{Study instrument and procedure}

The survey instrument included questions on the following domains: 1) Sources of information on the COVID-19 pandemic and their trustworthiness, rated on a 5-item Likert scale with a score ranging from 1 (Most trustworthy) to 5 (Not trustworthy); 2) Perceived awareness of COVID-19 related epidemiology and treatment; 3) Attitudes and perspectives on COVID-19 and its public health management; 4) Self-care; and 5) Sociodemographic characteristics.

The data were collected using a self-administered questionnaire utilizing the Google Forms platform (Supplementary file). The reliability of the questionnaire was acceptable (Cronbach's alpha $=0.623$ ). We adopted a snowball sampling method, with the survey weblink disseminated through various local instant messaging groups where membership was restricted to medical doctors.

\section{Operational definitions}

1. Perceived awareness - was considered adequate if the respondent reported being aware of these four items; the existing burden of disease, mode of transmission, appropriate PPE use guidelines, and the steps of PPE suit donning and doffing.
2. Perceived self-care - was considered adequate if the respondent reported receiving all of the following: adequate sleep and rest, having a healthy diet, and adherence to handhygiene guidelines at work.

3. Overall attitude - was measured by adding the scores of the following rating scales: perceived risk (individual), perceived risk (family), and their perceived outlook towards overcoming the virus.

\section{Statistical analysis}

The dataset was exported in the Microsoft Excel format, cleaned, and analyzed with IBM SPSS Version 25. The data were summarized as frequency and proportions for categorical variables, medians for ordinal data, and means with standard deviations for continuous data. The difference between proportions was assessed using the chi-squared test, and adjusted odds ratios at $95 \%$ confidence intervals are reported. A p-value $<0.05$ was considered statistically significant.

\section{RESULTS}

A total of 432 medical doctors responded to the survey, with 251 in the first round and 181 in the second round. The respondents comprised 227 (52.5\%) male and 205 $(47.5 \%)$ female doctors. The mean $( \pm \mathrm{SD})$ respondent age was $31( \pm 10.7)$ years. A total of $322(74.5 \%)$ respondents were from Northern India, 53 (12.3\%) from Southern India, $28(6.5 \%)$ from Eastern India, and 29 (6.7\%) from Western and Central India. By qualification, 250 (57.8\%) respondents were postgraduate (specialist) doctors, 92 (21.3\%) graduate MBBS doctors, and 90 (20.8\%) were medical intern trainees. Among the respondents, 173 (40\%) reported being directly involved in the management of suspected or confirmed COVID-19 cases. No significant difference in the characteristics of the survey respondents was observed across both rounds.

The respondents' interest in obtaining COVID-19 related information was high (median score $=4$ ), and it remained constant during both rounds of the survey. The extent of perceived trustworthiness of COVID-19 related information among the participants varied with its source (Table 1). Higher trustworthiness was reported for official government bulletins released by the Indian Council of Medical Research $(84.2 \%)$ or the Ministry of Health and Family Welfare (84.5\%), and least trustworthiness expressed for information circulated on social media networks (6.94\%), and this difference was not significant when comparing the responses from both rounds ( $\mathrm{p}=0.392$ ).

The perceived awareness of the respondents on aspects of COVID-19 epidemiology, prevention, and policy are reported in Table 2. A majority (52.8\%) of the respondents reported inadequate awareness but it was higher in those respondents involved in the management of COVID-19 patients $(\mathrm{p}=0.086)$ (Table 3).

A majority (52.3\%) of the respondents reported never 
Table 1. Perceived trustworthiness of COVID-19 information sources among medical doctors in India ( $\mathrm{N}=432$ )

\begin{tabular}{lc|cc|cc} 
Source & $\begin{array}{c}\text { Most trustworthy } \\
\mathbf{n}(\%)\end{array}$ & $\begin{array}{c}\text { More trustworthy } \\
\mathbf{n}(\%)\end{array}$ & $\begin{array}{c}\text { Trustworthy } \\
\mathbf{n}(\%)\end{array}$ & $\begin{array}{c}\text { Less trustworthy } \\
\mathbf{n}(\%)\end{array}$ & $\begin{array}{c}\text { Not trustworthy } \\
\mathbf{n}(\%)\end{array}$ \\
\hline ICMR & $131(30.3)$ & $100(23.1)$ & $133(30.8)$ & $55(12.7)$ & $13(3.0)$ \\
MoHFW & $136(31.5)$ & $96(22.2)$ & $133(30.8)$ & $53(12.3)$ & $14(3.2)$ \\
Print media & $172(39.8)$ & $28(6.5)$ & $17(3.9)$ & $172(12.0)$ & $163(37.8)$ \\
Online media & $12(2.8)$ & $56(13.0)$ & $176(40.7)$ & $150(34.7)$ & $38(8.8)$ \\
TV news & $14(3.2)$ & $41(9.5)$ & $26(6.0)$ & $142(32.9)$ & $110(25.5)$ \\
WhatsApp & $0(0)$ & $3(0.7)$ & $28(6.5)$ & $149(34.5)$ & $261(60.4)$ \\
Facebook & $0(0)$ & $2(0.5)$ & & & $253(58.6)$
\end{tabular}

ICMR: Indian Council of Medical Research. MoHFW: Ministry of Health and Family Welfare.

Table 2. Change in the perceived awareness and perspectives on COVID-19 management in Indian health settings among medical doctors in India, $2020(\mathrm{~N}=432)$

\begin{tabular}{|c|c|c|c|c|}
\hline Variable & \begin{tabular}{|c|} 
Perceived \\
awareness present \\
(3-9 April) \\
(n=251)
\end{tabular} & $\begin{array}{c}\text { Perceived } \\
\text { awareness present } \\
(18-24 \text { May }) \\
(\mathbf{n}=181)\end{array}$ & Total & $\mathbf{p}$ \\
\hline Perceived awareness & n (\%) & n (\%) & n (\%) & \\
\hline Burden of disease & $203(80.2)$ & $117(65.0)$ & $320(74.0)$ & $<0.001$ \\
\hline Mode of transmission & $225(88.9)$ & $155(86.1)$ & $380(88.0)$ & 0.382 \\
\hline PPE use guidelines & $232(92.4)$ & $149(82.3)$ & $381(88.2)$ & 0.002 \\
\hline PPE suit donning and doffing & $167(66.0)$ & $131(72.8)$ & $298(69.0)$ & 0.130 \\
\hline Testing protocol & $146(57.7)$ & $101(56.1)$ & $247(57.2)$ & 0.740 \\
\hline Home quarantine guidelines & $211(83.4)$ & $140(77.8)$ & $351(81.2)$ & 0.140 \\
\hline Self-isolation guidelines & $208(82.2)$ & $141(78.3)$ & $349(80.8)$ & 0.010 \\
\hline HCQ prophylaxis guidelines & $152(60.1)$ & $115(63.9)$ & $267(61.6)$ & 0.420 \\
\hline Risk perception & Mean (SD) & Mean (SD) & Mean (SD) & \\
\hline Individual risk perception & $3.5(1.13)$ & $3.6(1.05)$ & $3.5(1.1)$ & 0.308 \\
\hline Familial risk perception & $4.1(1.2)$ & $4.1(1.1)$ & $4.1(1.2)$ & 0.557 \\
\hline Overcoming the pandemic & $3.0(1.1)$ & $2.9(1.1)$ & $3.0(1.1)$ & 0.625 \\
\hline Perspectives on COVID-19 & Agree n (\%) & Agree n (\%) & Agree n (\%) & \\
\hline Indian ethnicity greater immunity & $120(47.4)$ & $105(58.3)$ & $225(52.1)$ & 0.025 \\
\hline High temperature inhibits virus & $76(30.0)$ & $58(32.2)$ & $134(31.0)$ & 0.625 \\
\hline BCG vaccine protective & $57(22.5)$ & $45(25.0)$ & $102(23.6)$ & 0.546 \\
\hline Yoga can augment immunity & $164(64.8)$ & $130(72.2)$ & $294(68.0)$ & 0.100 \\
\hline Alternative medicine protective & $82(32.4)$ & $66(36.7)$ & 148 (34.3) & 0.350 \\
\hline Masks for asymptomatic people & $147(58.1)$ & $166(92.2)$ & $313(72.4)$ & $<0.001$ \\
\hline
\end{tabular}

PPE: personal protective equipment. HCQ: hydroxychloroquine. BCG: Bacillus Calmette-Guerin.

having received training related to COVID-19 management protocols. Among those who had already received training $(\mathrm{n}=206), 118(57.5 \%)$ received it only once, 69 (33.6\%) received it on more than one occasion, and $40(19.5 \%)$ were regularly trained at least once a week.
Doctors experience considerable risk of infection when treating suspected or confirmed COVID-19 cases. In this study, the median perceived risk score of contracting COVID-19 disease among the respondents was 4 (maximum score $=5$ ), but the perceived threat of the transmission of 
Table 3. Factors associated with perceived awareness, attitude and self-care practices among medical doctors in India ( $=432)$

\begin{tabular}{|c|c|c|c|c|c|c|c|c|c|}
\hline Characteristic & Total & $\begin{array}{c}\text { Perceived } \\
\text { awareness } \\
\text { Adequate } \\
\text { n (\%) }\end{array}$ & AOR $(95 \% \mathrm{CI})$ & $\mathbf{p}$ & $\begin{array}{l}\text { Attitudes } \\
\text { mean } \\
\text { (SD) }\end{array}$ & $\mathbf{p}$ & $\begin{array}{l}\text { Self-care } \\
\text { practice } \\
\text { Adequate } \\
\text { n (\%) }\end{array}$ & AOR $(95 \% \mathrm{CI})$ & $\mathbf{p}$ \\
\hline Overall & 432 & 204 & & & $10.6(2.2)$ & & 277 & & \\
\hline \multicolumn{10}{|l|}{ Gender } \\
\hline Male & 227 & $119(52.4)$ & $1.6(1.1-2.3)$ & 0.017 & $10.8(2.0)$ & 0.017 & $147(64.7)$ & 1 & \\
\hline Female & 205 & $85(41.4)$ & 1 & & $10.4(2.0)$ & & $130(63.4)$ & $1.1(0.6-1.5)$ & 0.95 \\
\hline \multicolumn{10}{|l|}{ Age (years) } \\
\hline$<35$ & 332 & $160(48.2)$ & $1.2(0.72-1.99)$ & 0.47 & $10.5(2.0)$ & 0.076 & $201(60.5)$ & 1 & \\
\hline$\geq 35$ & 100 & $44(44.0)$ & 1 & & $10.9(2.0)$ & & $76(76.0)$ & $1.9(1.1-3.3)$ & 0.02 \\
\hline \multicolumn{10}{|l|}{ Qualification } \\
\hline Graduate/intern & 182 & $86(42.1)$ & 1 & 0.971 & $10.6(2.0)$ & 0.990 & $118(64.8)$ & $0.99(0.62-1.6)$ & 0.97 \\
\hline Specialist & 250 & $119(47.6)$ & $1.0(0.64-1.57)$ & & $10.6(2.0)$ & & $159(63.6)$ & 1 & \\
\hline \multicolumn{10}{|c|}{ COVID-19 patient care } \\
\hline Yes & 173 & $91(52.6)$ & $1.43(0.95-2.1)$ & 0.086 & $10.9(2.0)$ & 0.014 & $87(50.3)$ & 1 & $<0.001$ \\
\hline No & 259 & $113(43.6)$ & 1 & & $10.4(2.0)$ & & $190(73.4)$ & $2.6(1.6-4)$ & \\
\hline \multicolumn{10}{|l|}{ Training } \\
\hline Yes & 187 & $91(48.7)$ & $0.93(0.63-1.2)$ & 0.698 & $10.7(2.0)$ & & $116(62.0)$ & 1 & 0.68 \\
\hline No & 245 & 113 (46.1) & 1 & & $10.6(2.0)$ & 0.562 & 161 (65.7) & $1.1(0.72-1.6)$ & \\
\hline
\end{tabular}

AOR: adjusted odds ratio. SD: standard deviation.

COVID-19 disease to their family members was higher ( median $=5$ ). Nevertheless, both the perceived individual and familial risk did not significantly increase amongst the respondents in the second round despite rapid escalation in the COVID-19 disease burden of the country.

Access to adequate personal protective equipment was reported by only $45(17.8 \%)$ respondents during the first round, which increased to $104(57.7 \%)$ during the second round $(\mathrm{p}<0.001)$, suggesting increased domestic manufacturing and improved supply-chain logistics. Furthermore, among those respondents who had previously received training for COVID-19 in their institution, their perceived confidence of the correct knowledge of safely donning and doffing the PPE suit was significantly higher $(79.1 \%)$ compared to those who did not receive any training (59.3\%) ( $\mathrm{p}<0.001)$.

Most respondents expressed uncertainty regarding their views on aspects of COVID-19 epidemiology and management that lacked evidence or academic consensus. These included the likely effect of temperature and humidity on the viability of coronavirus transmission and the possible role of the Bacillus Calmette-Guerin (BCG) vaccine for protection against COVID-19 (Table 2). With regard to the recommendation for prophylaxis with the hydroxychloroquine drug, a total of 71 (39.2\%) agreed, 38
(21\%) were unsure, and 72 (39.8\%) respondents disagreed $(\mathrm{n}=181)$.

Many countries, including India, initiated stringent countrywide lockdowns to enforce social distancing, reduce crowded environments by shutting down schools and offices, and ceasing all modes of travel and transport, to inhibit the transmission of the virus. During the first round of the survey, most $(78.5 \%)$ respondents believed that the initially declared 21-day lockdown was inadequate for flattening the curve of the COVID-19 pandemic and a more prolonged period of lockdown was necessary. In the second round, the majority of the respondents considered that the ongoing lockdown implemented over eight weeks was effective in inhibiting the transmission of the virus (median score $=4$, maximum score $=5)$. A majority $(57.2 \%)$ also opined that the lockdown should be extended, while $14.4 \%$ were not sure $(n=181)$. Among those respondents who were against extension of the lockdown (28.7\%), the most common reasons cited were the perceived lack of further benefit in terms of reduction of the COVID-19 case burden (67.6\%), the harmful economic consequences (57.7\%), and the ineffective implementation of the lockdown by the administration (15.3\%).

During the first round of this study, most respondents felt the country's COVID-19 testing strategy was inadequate 
(68.5\%). However, confidence in the country's testing strategy significantly improved in the second round (56.4\%).

The outlook of the respondents towards the country successfully overcoming the COVID-19 pandemic was suboptimal (median score $=3$, maximum score $=5$ ), but the score did not significantly differ between the rounds $(\mathrm{p}=0.625)$.

Adherence to essential self-care practices for protection against COVID-19 were lacking in nearly $36 \%$ of the respondents. A binary logistic regression analysis was conducted to find associations of age, gender, academic qualification, involvement in COVID-19 patient care, and the prior COVID-19 training received with perceived awareness, attitudes and self-care practices of the participants. A significantly lower reporting of adherence to self-care practices was independently associated with direct involvement in the management of COVID-19 patients $(\mathrm{p}<0.001)$ and age $<35$ years $(\mathrm{p}=0.02)$ (Table 3$)$.

\section{DISCUSSION}

In the present study, most medical doctors reported a preference for obtaining information on COVID-19 from official government sources. A study in Pakistan among HCWs revealed reliance on sources other than government sources ${ }^{5}$. Most respondents perceived the COVID-19 testing rate in India to be insufficient, contrary to the government view, indicating the influence of media and non-government academic sources ${ }^{6,7}$. Similarly, a majority of the doctors were not convinced of the recommendation for hydroxychloroquine (HCQ) prophylaxis by the Indian Council of Medical Research for frontline workers, probably due to the lack of published evidence supporting its effectiveness ${ }^{8}$.

The perceived awareness of the respondents in our study was suboptimal, especially with regard to the appropriate use of PPE, suggesting the need for improvement and regularity in the training protocol. However, compared to the findings of a study among HCWs in China ${ }^{9}$, the risk perception of COVID-19 among doctors in the present study was lower. The Chinese study also reported the failure of HCWs to maintain quarantine with their families, thereby increasing the inadvertent spread of the coronavirus infection ${ }^{9}$. Consequently, administrations should prioritize the provision of safe residential lodgings for doctors away from their homes and families during a designated quarantine period, which is a challenge in resource-limited settings.

Pandemics reflect an unprecedented challenge for public health systems, especially the health workers involved in the provision of care to patients. The morale of the healthcare workers needs to be sustained during a pandemic expected to last for at least 12 to 18 months $^{10}$. In this study, the self-efficacy of the doctors was poor, and significantly lower among those in the frontline $(p<0.001)$, signifying the need for public health initiatives enabling provision of care and support for this valuable but vulnerable group.

\section{Limitations}

The respondents were limited to those having access to the internet and social media. Training sessions, testing protocols, and the availability of PPE constitute a dynamic phenomenon that needs repeated assessment during the ongoing pandemic. There was also an overrepresentation of participants from the northern Indian states in the survey, thus restricting its generalizability. Finally, significant disparities exist between various Indian states with respect to the rate of spread of the disease, development of hotspots, available testing capacity and tests conducted, healthcare system preparedness, etc. However, an inter-state comparison was not possible due to the smaller number of participants from states other than Delhi.

\section{CONCLUSIONS}

Despite a high level of interest for COVID-19 related information among medical doctors in India, critically significant gaps exist in their awareness of certain local aspects of COVID-19 management. Training deficiencies were also reported, which constitutes a potential occupational risk hazard. The perspective of the doctors on public health aspects of COVID-19 management reflected heterogeneous stances, although it was aligned with the academic consensus in a majority of instances. Most doctors shared a direct medicalized perspective, as opposed to the negative economic considerations, in supporting the implementation of a nationwide lockdown.

\section{REFERENCES}

1. COVID19 India Website. https://www.covid19india.org/. Accessed October 10, 2020.

2. Roy D, Tripathy S, Kar SK, Sharma N, Verma SK, Kaushal V. Study of knowledge, attitude, anxiety \& perceived mental healthcare need in Indian population during COVID-19 pandemic. Asian J Psychiatr. 2020;51:102083. doi:10.1016/j.ajp.2020.102083

3. O'Sullivan ED. PPE guidance for covid-19: be honest about resource shortages. BMJ. 2020;369:m1507. doi:10.1136/bmj.m1507

4. COVID-19: protecting health-care workers. Lancet. 2020;395(10228):922. doi:10.1016/S0140-6736(20)30644-9

5. Saqlain M, Munir MM, Rehman SU, et al. Knowledge, attitude, practice and perceived barriers among healthcare workers regarding COVID-19: a cross-sectional survey from Pakistan. J Hosp Infect. 2020;105(3):419-423. doi:10.1016/j.jhin.2020.05.007

6. Vaidyanathan G. People power: How India is attempting to slow the coronavirus. Nature. 2020;580(7804):442. doi:10.1038/d41586-020-01058-5

7. Indian Council of Medical Research, Department of Health Research. Strategy of COVID-19 testing in India. https:// www.mohfw.gov.in/pdf/LabTestingAdvisory.pdf. Published March 17, 2020. Accessed May 29, 2020.

8. Indian Council of Medical Research, Department of Health Research. Revised advisory on the use of 
Hydroxychloroquine (HCQ) as prophylaxis for COVID-19 infection (in supersession of previous advisory dated 23rd March, 2020). https://www.mohfw.gov.in/pdf/ RevisedadvisorychloroquineasprophylaxisforSARSCOVID19 infection.pdf. Accessed May 29, 2020.

9. Zhang M, Zhou M, Tang F, et al. Knowledge, attitude, and practice regarding COVID-19 among healthcare workers in Henan, China. J Hosp Infect. 2020;105(2):183-187. doi:10.1016/j.jhin.2020.04.012

10. Mishra P, Bhadauria US, Dasar PL, et al. Knowledge, attitude and anxiety towards pandemic flu a potential bio weapon among health professionals in Indore City. Przegl Epidemiol. 2016;70(1):41-45, 125-127. PMID:27344473.

\section{ACKNOWLEDGMENTS}

We thank the participants for their valuable time in responding to the survey.

\section{CONFLICTS OF INTEREST}

The authors have completed and submitted the ICMJE Form for Disclosure of Potential Conflicts of Interest and none was reported.

\section{FUNDING}

There was no source of funding for this research.

PROVENANCE AND PEER REVIEW

Not commissioned; externally peer reviewed. 